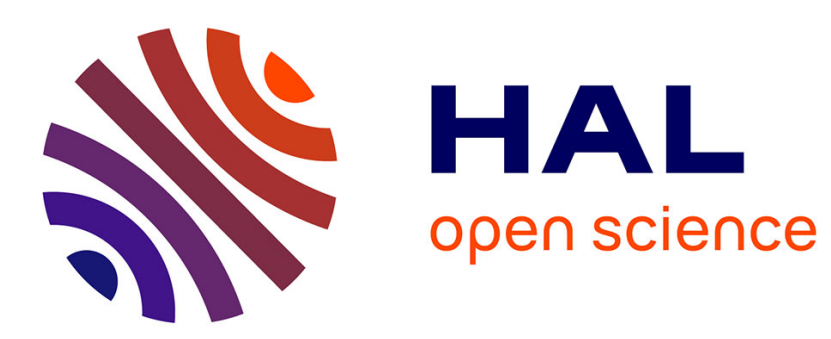

\title{
Measurements and time-domain simulations of multiphonics in the trombone.
}

\author{
Lionel Velut, Christophe Vergez, Joël Gilbert
}

\section{To cite this version:}

Lionel Velut, Christophe Vergez, Joël Gilbert. Measurements and time-domain simulations of multiphonics in the trombone.. Journal of the Acoustical Society of America, 2016, 140 (4), pp.2876. 10.1121/1.4964634. hal-01363547v2

\section{HAL Id: hal-01363547 \\ https://hal.science/hal-01363547v2}

Submitted on 4 Nov 2016

HAL is a multi-disciplinary open access archive for the deposit and dissemination of scientific research documents, whether they are published or not. The documents may come from teaching and research institutions in France or abroad, or from public or private research centers.
L'archive ouverte pluridisciplinaire HAL, est destinée au dépôt et à la diffusion de documents scientifiques de niveau recherche, publiés ou non, émanant des établissements d'enseignement et de recherche français ou étrangers, des laboratoires publics ou privés. 


\title{
Measurements and time-domain simulations of multiphonics in the trombone.
}

\author{
Lionel Velut ${ }^{1}$, Christophe Vergez ${ }^{1}$, and Joël Gilbert ${ }^{2}$ \\ ${ }^{1}$ LMA, CNRS, UPR 7051, Aix-Marseille Univ., Centrale Marseille, F-13453 \\ Marseille cedex 13, France. \\ ${ }^{2}$ Laboratoire d'Acoustique de l'Université du Maine, UMR CNRS-6613, Avenue \\ Olivier Messiaen, 72085 Le Mans cedex 9, France
}

November 4, 2016

\begin{abstract}
Multiphonic sounds of brass instruments are studied in this article. They are produced by playing a note on a brass instrument while simultaneously singing another note in the mouthpiece. This results in a peculiar sound, heard as a chord or a cluster of more than two notes in most cases. This effect is used in different artistic contexts.

Measurements of the mouth pressure, the pressure inside the mouthpiece and the radiated sound are recorded while a trombone player performs a multiphonic, firstly by playing an $F_{3}$ and singing a $C_{4}$, then playing an $F_{3}$ and singing a note with a decreasing pitch. Results highlight the quasi-periodic nature of the multiphonic sound and the appearance of combination tones due to intermodulation between the played and the sung sound.

To assess the ability of a brass instrument physical model to reproduce the measured phenomenon, time-domain simulations of multiphonics are carried out. A trombone model consisting in an exciter and a resonator non-linearly coupled is forced while self-oscillating to reproduce simultaneous singing and playing. Comparison between simulated and measured signals is discussed. Spectral content of the simulated pressure match very well with the measured one, at the cost of a high forcing pressures.
\end{abstract}

\section{INTRODUCTION}

A "monodic instrument", by its etymological meaning, is an instrument designed to play "monophonic" sounds, that is sounds with a single pitch. However, most monodic instruments of the orchestra can also produce unusual sounds, called "multiphonic sounds". This term means that the listener perceives multiple notes in the sound.

A range of wind instruments can produce sounds called multiphonics [Castellengo, 1981]. Actually, this word designates two distinct phenomena. On the one hand, the term "multiphonic" can refer to a multiple-pitched sound generated with an extension of the conventional playing techniques: woodwind multiphonics, where quasi-periodic regimes are generated through specific embouchures and/or fingerings [Backus, 1978, Keefe and Laden, 1991, Dalmont et al., 1995, Gibiat and Castellengo, 2000, Doc et al., 2014], belong to this category. A quasi-periodic oscillation is a deterministic oscillation whose energy is located at frequencies which are integer combinations of base frequencies. The ratios between the base frequencies are irrational 
numbers. Flute-like instrument multiphonics are also based on an extended playing technique: the oscillation of the air jet becomes quasi-periodic with no need to introduce another oscillator [Campbell and Greated, 1994, Blanc et al., 2010, Terrien et al., 2013]. Brass instrument multiphonics can be based on two distinct mechanisms: a spontaneous quasi-periodic self-oscillation, similar to those in flutes and in reed instruments, can be involved [Castellengo et al., 1983]. But on the other hand, the brass instrument player can also produce a multiphonic sound by singing while he plays [Campbell and Greated, 1994, Sluchin, 1995]: two oscillators are then involved, the lips and the vocal folds. This paper only focuses on this latter kind of multiphonic sound on the trombone. In this situation, an intermodulation is observed, making non-harmonic frequencies appear in the resulting sound.

According to the musical context, a multiphonic sound can be considered as a mistake made by the musician or due to a defect of the instrument: in occidental art-music, a "rolling sound" is generally considered to be a mistake the musician makes and a bowed instrument with "wolf notes" [Iniacio et al., 2008] is considered to be of poor quality. However, multiphonic sounds can also be intentional. This applies to extra-European traditional instruments such as pre-Columbian flautas de chinos producing sonidos rajados [Wright and Campbell, 1998, Blanc et al., 2010, Terrien et al., 2013], or the Australian aboriginal didjeridu [Wolfe and Smith, 2008] where the musician has several options for modifying the sound by singing or tuning of vocal tract resonance while he plays. But European classical music also includes examples of multiphonics: an example of cadenza of a French horn concertino is displayed in the score in Figure 1. The technique is mentioned in teaching methods of the $19^{\text {th }}$ century, as reported in [Sluchin, 1995]. Jazz and avant-garde music have popularized this musical effect with artists like James Morrison, Nils Landgren, Nat McIntosh and many others.

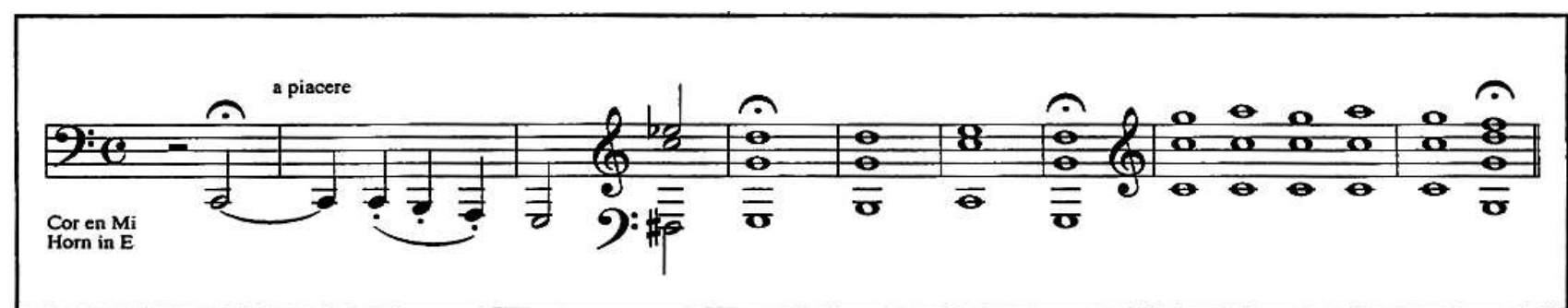

Figure 1: Cadenza from C.M. von Weber's concertino for horn, including multiphonic sounds.

Multiphonics produced by a musician simultaneously singing and playing a brass instrument have been documented in [Campbell and Greated, 1994, Sluchin, 1995] but, to the author's knowledge, simulations of this phenomenon have never been carried out. This paper examines the ability of a simple instrument physical model to simulate trombone multiphonics. This helps a better understanding of the multiphonic phenomenon and defines the abilities of the considered model. Comparisons between results of this model and measurements on trombone players are proposed on both multiphonic sounds studied: namely, playing an $F_{3}$ while singing a $C_{4}$ (referred to hereafter as $F_{3}-C_{4}$ multiphonic), and playing an $F_{3}$ while singing a note whose pitch decreases from $C_{4}$ to slightly above $C_{3}$ (referred to later as "decreasing playing frequency multiphonic"). The measurement and simulation tools are first presented in Section 2; then, measurements and simulations of a selection of multiphonic sounds are compared in Section 3 to evaluate the ability of the model to reproduce multiphonics. 


\section{TOOLS}

\section{II.A Experimental setup}

An experimental device has been developed to measure some characteristic variables during a trombone multiphonic performance. The acoustic pressure inside the instrument mouthpiece $p(t)$, characteristic of the response of the instrument, is measured. Another sensor measures the blowing pressure as well as the acoustic pressure inside the mouth $p_{m}(t)$. The radiated sound, $p_{\text {ext }}(t)$, is also recorded. The measurements room has a short reverberation time, similar to that of a rehearsal studio, for the musician's convenience.

- The mouth pressure $p_{m}(t)$ is measured with an Endevco 8510B-5 miniature pressure sensor, through a capillary tube $(1.5 \mathrm{~mm}$ inner diameter) inserted in the mouth of the musician. The capillary tube is glued to a short piece of silicone tubing (4 mm diameter) connected to the pressure sensor. The assembly of tubes forms a Helmholtz resonator which bandpassfilters the signal. An ad hoc convolution filter is applied to the measured signal in order to compensate for the transfer function of the tube.

- The pressure inside the mouthpiece $p(t)$ is measured through the same sensor model. The sensor is screwed into the mouthpiece wall. The microphone is small enough for the shape of the mouthpiece cup not to be significantly altered.

- The radiated sound $p_{\text {ext }}(t)$ is recorded with a $B \& K 1 / 4$ " microphone, placed about $40 \mathrm{~cm}$ downstream from the center of the trombone's bell. The vicinity with the bell limits the influence of the reflections in the room.

These sensors are connected to their respective conditioners/amplifiers. The signals are simultaneously recorded by a computer through NI-9234 and 9215 acquisition modules. The sampling frequency is $51200 \mathrm{~Hz}$.

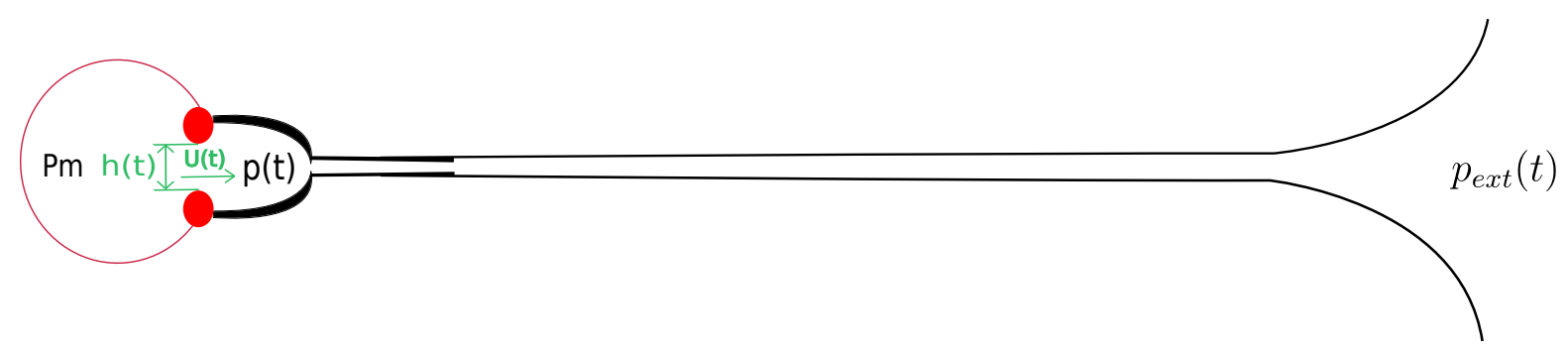

Figure 2: (color online) Sketch of the trombone positioning $p_{m}(t), p(t)$ and $p_{\text {ext }}(t)$. Some variables of the model defined in Section II.B are written in green.

\section{II.B Time-domain simulation of a trombone's physical model}

All along this article, measurements are compared with time-domain simulations from a trombone physical model. The retained self-sustained model relies on a linear exciter which is non-linearly coupled with a linear resonator. Each of these three elements is described below. This kind of model has been widely used for wind instruments [Fletcher, 1993] including brass instruments [Eliott and Bowsher, 1982, Yoshikawa, 1995, Cullen et al., 2000], since the seminal work of von Helmoltz [von Helmholtz, 1877].

For brass instruments, the exciter consists of the lips of the musician, represented by a linear, oscillator-like valve, linking the height between the lips $h(t)$ and the pressure difference across the 
lips $\delta p(t)=p_{m}(t)-p(t)$. A one degree of freedom valve (referred to hereafter as "1-DOF valve") [Fletcher, 1993] is enough to model the lips for common playing situations [Yoshikawa, 1995] with a tractable number of parameters. Two kinds of 1-DOF valves can be considered : "striking outward", which tends to open when $\delta p$ grows, and "striking inward" which presents the opposite behavior. For the reasons detailed in [Velut et al., 2016] we chose a "striking outward" valve to model the lips of the trombonist. This model relies on the following equation:

$$
\frac{d^{2} h}{d t^{2}}+\frac{\omega_{l}}{Q_{l}} \frac{d h}{d t}+\omega_{l}^{2}\left(h-h_{0}\right)=\frac{1}{\mu}\left(p_{m}-p(t)\right),
$$

where $\omega_{l}=2 \pi f_{l}(\mathrm{rad} / \mathrm{s})$ is the lip resonance angular frequency; $Q_{l}$ the (dimensionless) quality factor of the lips; $h_{0}$ the value of $h(t)$ at rest $(\mathrm{m}) ; \mu$ an equivalent surface mass of the lips $\left(\mathrm{kg} \cdot \mathrm{m}^{-2}\right)$.

Lip parameters very similar to those chosen in [Velut et al., 2016] after a thorough bibliographical review are used. These parameters are given in Table I. The only difference is the quality factor $Q_{l}$ lessened from 7 to 5 . Results previously obtained in [Velut et al., 2016] were satisfactory, with reasonable threshold blowing pressures in the $[1 \mathrm{kPa}: 15 \mathrm{kPa}]$ range for commonly played notes. The refining of the $Q_{l}$ value results in periodic playing on a larger range of $f_{l}$ on the Jupiter JSL 2321 trombone used in the experiment.

\begin{tabular}{|c|c|c|c|}
\hline$h_{0}(\mathrm{~m})$ & $L(\mathrm{~m})$ & $1 / \mu\left(\mathrm{m}^{2} \mathrm{~kg}^{-1}\right)$ & $Q_{l}$ \\
\hline $5.10^{-4}$ & $12.10^{-3}$ & 0.11 & 5 \\
\hline
\end{tabular}

Table I: Lip parameters retained for this article.

In most studies about brasswinds, $p_{m}$ is assumed to be constant in usual playing, corresponding to the blowing pressure. However, in order to simulate a multiphonic, a forcing component is added to the static value of $p_{m}$, corresponding to the acoustic pressure produced by the vibrating vocal folds. For multiphonic simulations with a constant singing frequency, the $p_{m}$ signal takes the following form:

$$
p_{m}(t)=p_{m}^{0}+p_{m}^{1} \cdot \sin \left(2 \pi t f_{s}\right),
$$

where $p_{m}^{0}$ and $p_{m}^{1}$ are respectively the constant blowing pressure and the amplitude of the singing signal, and $f_{s}$ the singing frequency.

For simulations with a decreasing frequency, the signal is divided into 3 parts: first, the mouth pressure is a constant blowing pressure for 6 seconds: this gives time to reach a steady-state oscillating regime. Then, the $p_{m}$ signal is similar to Eq. (2) for 6 seconds with $f_{s}=f_{1}$. Then, the frequency decreases from $f_{1}$ to $f_{2}$ taking this form:

$$
p_{m}(t)=p_{m}^{0}+p_{m}^{1} \cdot \sin \left[2 \pi t\left(f_{s}+\frac{\left(f_{2}-f_{s}\right) \cdot t}{2 d}\right)\right],
$$

where $d$ is the duration of the frequency decrease $(\mathrm{s})$.

In the measurements, the $p_{m}^{1} / p_{m}^{0}$ ratio is about 0.02 , but higher values are used in simulations, to get computed spectra as close as possible to experimental ones. A value of $p_{m}^{1}=0.3 p_{m}^{0}$ is used all along this paper. This difference in $p_{m}^{1}$ values between simulation and measurement is a limitation of our model.

This exciter is non-linearly coupled to a resonator: the bore of the trombone. Propagation in brass instruments, particularly the trombone, is known to be nonlinear for loud tones. This causes nonlinear distortion of the waveform, possibly leading to shockwaves [Hirschberg et al., 1996, 
Myers et al., 2012]. In musical terms, the sound becomes "brassy" at louder playing dynamics. However, since this study focuses on low and moderate playing dynamics, a linear model of propagation is sufficient. Thus, the resonator is modeled by its input impedance $Z$. By definition, $Z$ is the ratio, in the frequency domain, of the pressure at the input of the resonator $P(\omega)$ to the acoustic flow $U(\omega)$ taken at this same point :

$$
Z(\omega)=\frac{P(\omega)}{U(\omega)} .
$$

The input impedance of the Jupiter JSL 2321 tenor trombone used for experiments (with the slide fully pulled in) is measured with the impedance sensor described in [Macaluso and Dalmont, 2011], then fitted by a sum of 13 Lorentzian functions -representing the 13 first modes of the tromboneusing a least squares method similar to that in [Silva, 2009].

The coupling between this resonator and the aforementioned exciter is non linear. It is provided by the airflow through the lip channel. The air jet is assumed to be laminar in the lip channel, then turbulent in the mouthpiece, all its kinetic energy being dissipated without pressure recovery. Applying the Bernoulli law and the mass conservation law between the mouth and the lip channel gives the following expression of the flow between the lips, depending on the pressure difference and the height of the lip channel [Eliott and Bowsher, 1982, Hirschberg et al., 1995]:

$$
u(t)=\sqrt{\frac{2}{\rho}} \cdot L \cdot h(t) \cdot \operatorname{sign}\left(p_{m}-p(t)\right) \cdot \sqrt{\left|p_{m}-p(t)\right|} \cdot \theta(h),
$$

where $u(t)$ is the airflow rate $\left(\mathrm{m}^{3} \cdot \mathrm{s}^{-1}\right), \rho=1.19 \mathrm{~kg} \cdot \mathrm{m}^{-3}$ the air density at $20{ }^{\circ} C, \theta(h)$ the Heaviside step function related to $h(t)$ and $L$ the width of the lip channel $(\mathrm{m})$.

Simulations based on this model are carried out with MoReeSC [Silva, 2013]. This open-access Python library solves the equations of the model numerically, based on the modal decomposition of the pressure signal in the instrument. This provides values of $p$, the lip opening $h$ and the airflow between the lips $u$ at each time sample. It features the possibility of modifying input parameters during the simulation, which is particularly useful in this study for defining time-varying mouth pressure signals. In order to get a simulated pressure, a measurement of the transfer function of the trombone, between $p$ and $p_{e x t}$ is made. Filtering the simulated $p$ with the given transfer function results in a simulated $p_{\text {ext }}$.

\section{II.C Preliminary measurement on vocal folds}

A preliminary experiment is carried out to assess the choice of a forcing term to model the singing like in Eq. (2) and (3). The production of a multiphonic sound requires two exciters: the lips of the musician and his vocal folds. In order to evaluate the independence of the vocal fold oscillation with respect to the lip oscillation, an estimation of the vocal fold oscillation is carried out by measuring the electrical conductivity of the glottis, similarly to what was done in the experimental campaign conducted on the didjeridu [Wolfe and Smith, 2008]. The glottis conductivity is assumed to be approximatively proportional to the contact area of the lips [Hezard et al., 2014]. Since we are interested in comparing orders of magnitude, this approximative proportionality is sufficient. An electroglottograph from VoceVista is used to measure the conductivity of the vocal folds while the musician plays an $F_{3}-C_{4}$ multiphonic. The spectrum of the resulting signal is shown in Fig. 3. 


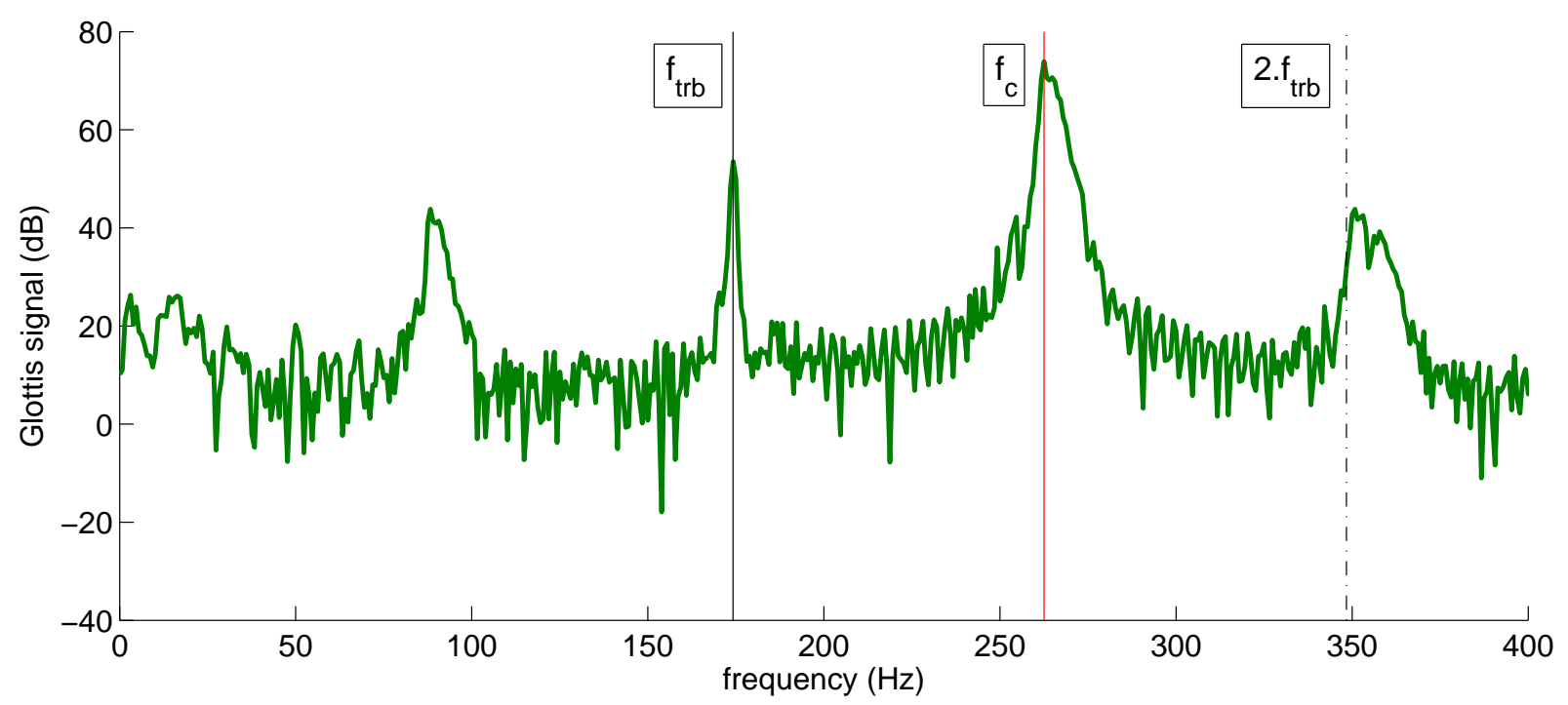

Figure 3: (color online) Spectrum of glottis signal when playing an $F_{3}-C_{4}$ multiphonic. Vertical lines indicate the playing frequency $f_{t r b}$ (black), the singing frequency $f_{s}$ (red) and $f_{\text {trb }}$ first harmonic (black, dash-dotted). Hanning window of width $0.2 \mathrm{~s}$, zero-padding of the signal until frequency precision is under $1 \mathrm{~Hz}$.

Spectral components can be observed at the singing frequency $f_{s}=262.5 \mathrm{~Hz}$ but also at the playing frequency $f_{\text {trb }}=174.2 \mathrm{~Hz}$, showing a coupling between the lips and the vocal folds. However, the amplitude of the $f_{t r b}$ component is $20 \mathrm{~dB}$ lower than the amplitude of the $f_{s}$ one: this indicates that the oscillation of the vocal folds is not much altered by the acoustic feedback. Thus, since we are interested in identifying the simplest model simulating multiphonic sounds, modeling the contribution of vocal folds through a forcing term seems to be a decent approximation, much simpler than a model that would take into account the vocal folds, the vocal tract and the lips . However, a time-domain simulation tool which would simulate the coupling with the impedance of the vocal tract would probably be of some interest. The implementation of such a model could be realized with the tools presented here.

\section{RESULTS}

\section{III.A $\quad F_{3}-C_{4}$ multiphonic}

\section{III.A.1 Experiment}

The study firstly focuses on the $F_{3}-C_{4}$ multiphonic, which is one of the most commonly played by trombonists and proposed as a first exercise in [Sluchin, 1995]. Producing an $F_{3}-C_{4}$ multiphonic consists in playing an $F_{3}$ while singing a $C_{4}$, i.e. a fifth above. In physical terms, this means playing on the third register of the trombone, while simultaneously singing the note whose frequency is 1.5 times higher $\left(f_{s} / f_{\text {trb }}=1.5\right)$. The musician is asked to successively sing a $C_{4}$, then play an $F_{3}$, then perform an $F_{3}-C_{4}$ multiphonic.

Figure 4 shows the spectrograms corresponding to this experiment and calculated for the time-domain signals of $p_{m}$ in Fig. 4 (a), $p$ in Fig.4 (b) and $p_{\text {ext }}$ in Fig.4 (c). The successive tasks - singing, playing, multiphonic - successively appear on the spectrograms. During the singing, a component appears at $f_{s}=259.8 H z$ with its harmonics. Similarly, $f_{t r b}=173.6 \mathrm{~Hz}$ and its 
harmonics appear while the musician is playing an $F_{3}$. A component at $f_{\text {trb }}$ can be observed in Fig. 4 (b) and (c) but also in the $p_{m}$ spectrogram, because of the coupling with the vocal tract of the musician [Chen et al., 2012, Fréour and Scavone, 2013]. When the multiphonic is played, $p(t)$ and $p_{\text {ext }}(t)$ contain the fundamental and harmonics of both $f_{s}$ and $f_{t r b}$. In addition, other frequency components also appear, which do not belong to either the harmonic series of $f_{s}$ or that of $f_{t r b}$. These components are shown by arrows in Fig. 4 (b). Note that one of these components has its frequency under $f_{t r b}$. Figure 5 superimposes the spectra of $p(t)$ during the three phases of the performance: singing, playing, multiphonic. This highlights that some peaks of the multiphonics spectrum clearly do not belong to the played signal or to the sung signal.

\section{III.A.2 Simulation}

This experiment (playing an $F_{3}$ on a trombone while singing a $C_{4}$ ) is simulated, using the method described in Section II.B with the parameters given in Table I: the physical model of trombone is set to play an $F_{3}$, on its $3^{d}$ register, with a lip resonance frequency $f_{l}=140 \mathrm{~Hz}$ and a steady blowing pressure set to $p_{m}^{0}=4500 \mathrm{~Pa}$. This value is slightly above the threshold pressure calculated by linear stability analysis, as in [Velut et al., 2016]. Then, the "sung" note is included to simulate the multiphonic: a forcing sinusoidal component is added to the static blowing pressure, at a frequency $f_{s}$ corresponding to the upper fifth, as written in Eq. (2). The amplitude of the forcing sinusoidal component is set to $30 \%$ of $p_{m}^{0}$ so that $p_{m}^{1}=1350 \mathrm{~Pa}$. This forcing component starts 3 seconds after the blowing pressure, once the self-oscillation of the instrument model has reached its steady state. This avoids interferences between the forcing component and the transitory phase of the self-sustained oscillation.

Spectrograms of the simulation results for $p_{m}, p$ and $p_{\text {ext }}$ are displayed in Fig. 6 (a), (b) and (c), respectively. First, the model auto-oscillates on its own until $t=3 \mathrm{~s}$; then the forcing component is added. Fig. 6 (a) does not display any spectral component at $f_{t r b}$, because the retained model does not take the coupling with the vocal tract into account. Before $t=3 s$, Fig. 6 (b) and (c) only display spectral components at $f_{t r b}=189 \mathrm{~Hz}$ and its harmonics. The oscillation frequency of the simulation is higher than the experimentally recorded playing frequency in Fig. 4. This is consistent with a well-known limitation of this brass model, known to oscillate at sharper frequencies than the tempered scale notes [Campbell, 2004, Silva et al., 2007, Chaigne and Kergomard, 2016, p.547]. Then, at $t=3 \mathrm{~s}$, the forcing component at $f_{s}=282.7 \mathrm{~Hz}$ appears. As in the experiment, $p(t)$ and $p_{\text {ext }}(t)$ show frequency components which are neither $f_{s}$, nor $f_{t r b}$, nor their harmonics. This is also to be seen in the $p$ spectra displayed in Fig. 7: some peaks of the multiphonic signal do not match $f_{s}, f_{t r b}$ (solid lines) or their harmonics (dashed lines). 

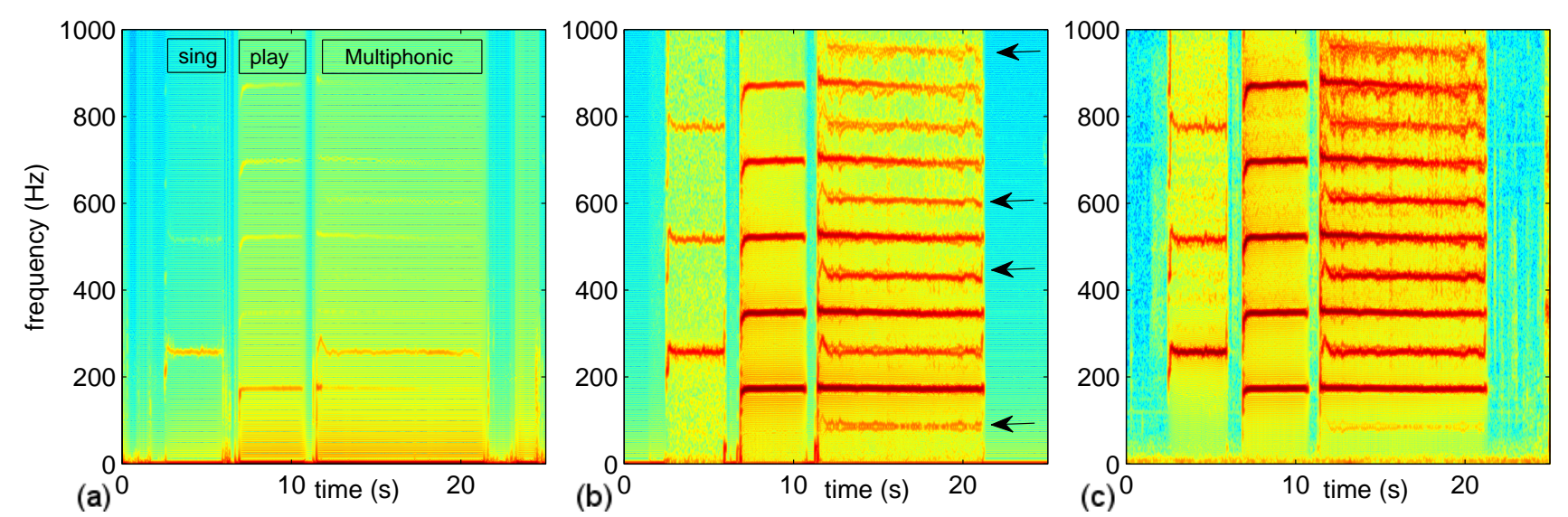

Figure 4: (color online) Experiment: spectrograms of the pressures in the mouth $p_{m}$ (a), in the mouthpiece $p(\mathrm{~b})$ and radiated $p_{\text {ext }}(\mathrm{c})$ measured in vivo. Hanning window of width $0.2 \mathrm{~s}$, 95\% overlap, zero-padding of the signal until frequency precision is under $1 \mathrm{~Hz}$. The musician successively sings $C_{4}(2.5-6.5 s)$, plays $F_{3}(7-11 s)$, then performs an $F_{3}-C_{4}$ multiphonic $(12-21 s)$. During multiphonic, the $p$ and $p_{\text {ext }}$ spectrograms exhibit spectral components which do not belong to either the sung or the played note: they are designated with arrows in (b).
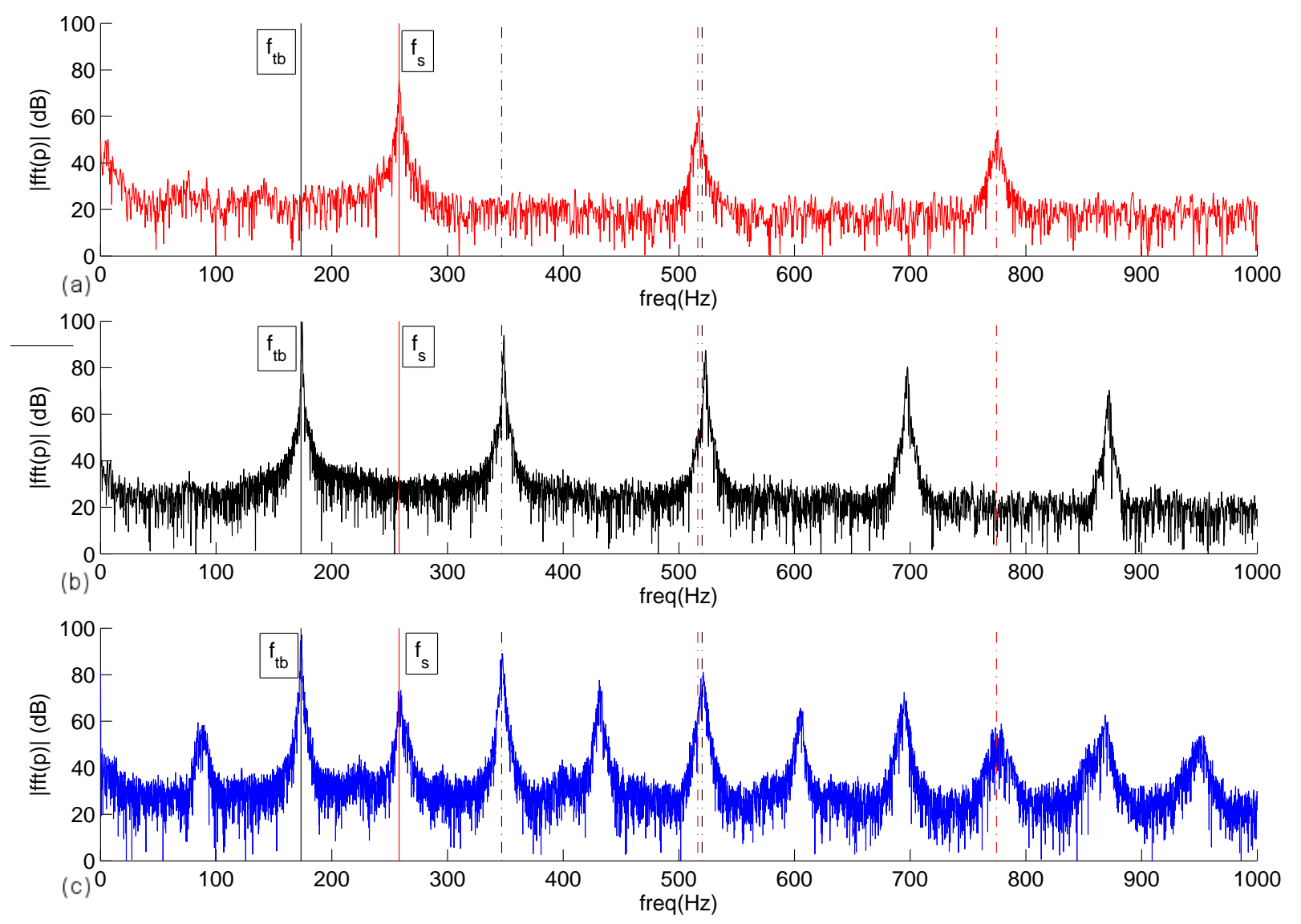

Figure 5: (color online) Experiment: spectra of the mouthpiece pressure $p(t)$ from the same performance: playing (a), singing (b), multiphonic (c). Spectra taken from the spectrograms in Fig. 4b). Peaks appear in the multiphonic spectrum, whose frequencies match neither the oscillation frequency $f_{t r b}=173.6 \mathrm{~Hz}$ nor the singing frequency $f_{s}=259.8 \mathrm{~Hz}$ nor their harmonics. $f_{\text {trb }}$ and $f_{s}$ are represented as vertical plain lines (fundamentals) and dash-dotted lines (harmonics). 

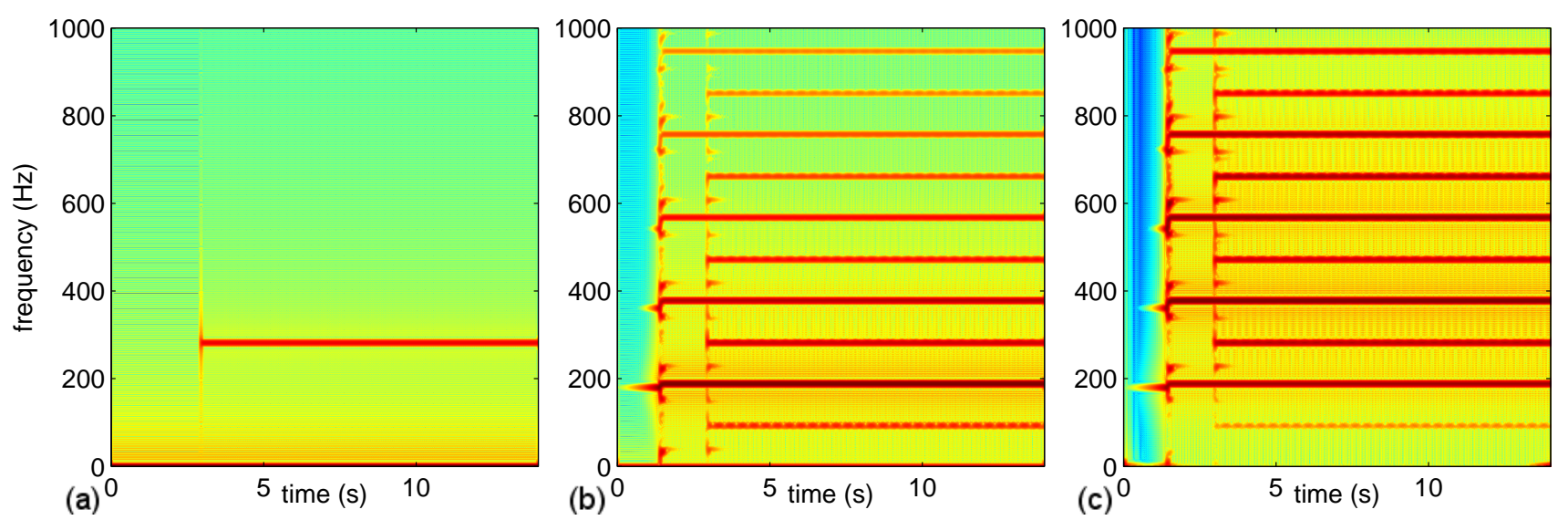

Figure 6: (color online) Simulation: spectrograms of the simulated pressures in the mouth $p_{m}$ (a), in the mouthpiece $p(\mathrm{~b})$ and radiated $p_{\text {ext }}(\mathrm{c})$. Hanning window of width $0.2 \mathrm{~s}$, overlapping of $95 \%$, zero-padding of the signal until frequency precision is under $1 \mathrm{~Hz}$. The blowing pressure $p_{m}$ has a stationary component $\left(p_{m}^{0}=4500 \mathrm{~Pa}\right)$ and, after $t=3 \mathrm{~s}$, an oscillating component (amplitude $p_{m}^{1}=1350 \mathrm{~Pa}$, frequency $f_{s}=282.7 \mathrm{~Hz}$ ). The transient occurs at $t=1.3 \mathrm{~s}$, the forcing signal is added after $t=3 \mathrm{~s}$. As in the experiment, spectral components other than harmonics of the playing and the singing frequencies appear.

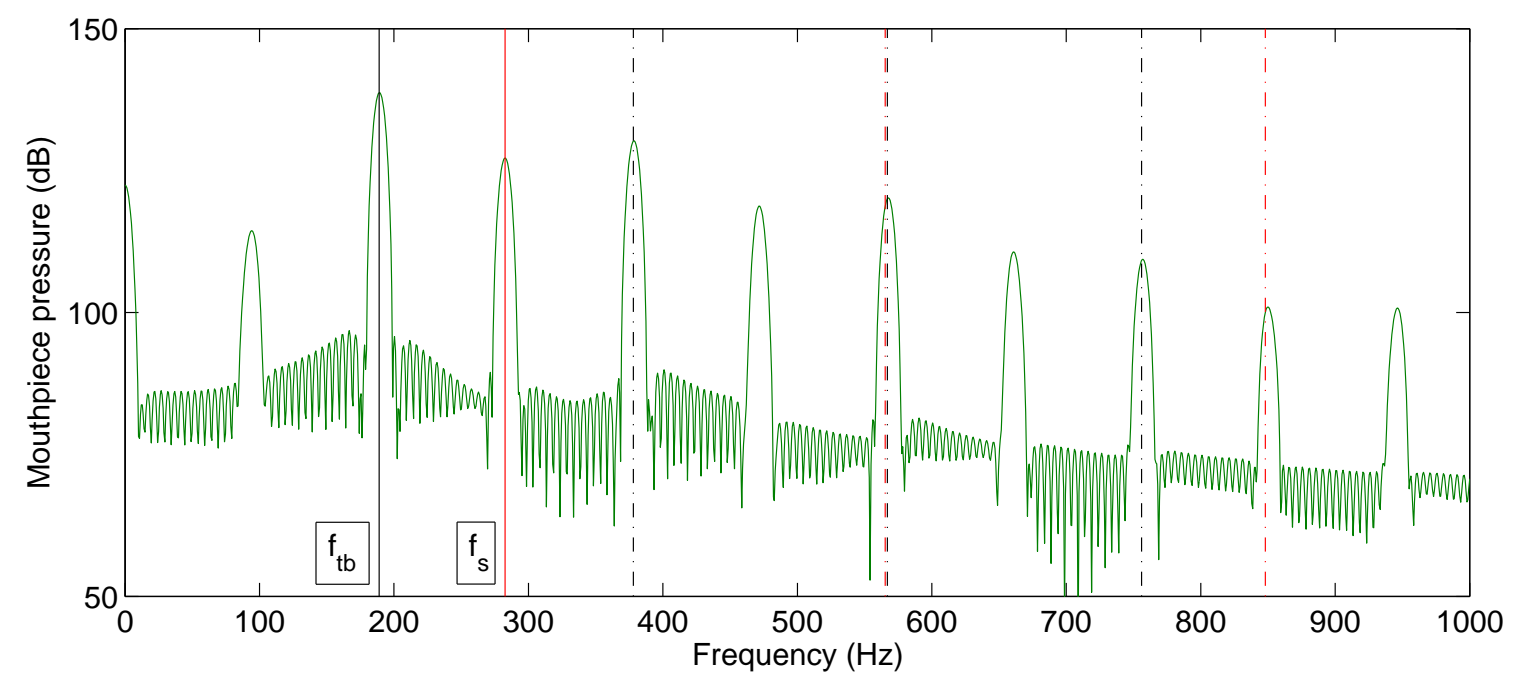

Figure 7: (color online) Simulation: spectrum of the mouthpiece pressure $p(t)$ from the simulation of the multiphonics. Spectrum is taken from spectrogram in Fig. 6b). $f_{t r b}=189 \mathrm{~Hz}, f_{s}=282.7 \mathrm{~Hz}$ and their harmonics are shown. Some frequencies are neither harmonic of $f_{s}$ nor of $f_{\text {trb }}$ but are integer combinations of those.

\section{III.A.3 Discussion}

Frequencies of the peaks appearing in the spectra of multiphonics, either simulated (Fig. 6 and 7) or measured(Fig. 4 and 5), match very well integer combinations of $f_{s}$ and $f_{\text {trb }}$ : the relative error is less than $3 \%$ for the measured frequencies and less than $0.5 \%$ for the simulated frequencies. Table II reports the frequencies appearing in the simulation and in the measurement, and proposes one or two integer combinations giving the same frequency. A given integer combination matches the peak of the same rank in the experiment and in the simulation. These frequency components at integer combinations of $f_{\text {trb }}$ and $f_{s}$ are hereafter called combination tones (CT), 
as in [Campbell and Greated, 1994, p.64]. In terms of amplitude, the peaks in the spectra of the simulated and the measured multiphonics are not the same, even if the shapes of the spectral envelopes remain comparable.

\begin{tabular}{|c||c|c|c|c|c|c|c|c|c|c|}
\hline Peak No. & 1 & 2 & 3 & 4 & 5 & 6 & 7 & 8 & 9 & 10 \\
\hline$f_{\text {exp }}(H z)$ & 86.1 & 176 & 259.8 & 347.3 & 433.4 & 521 & 605.1 & 694.8 & 778.7 & 868.7 \\
\hline$f_{\text {sim }}(H z)$ & 94.15 & 189 & 282.7 & 377.9 & 471.4 & 567.6 & 661.1 & 756.6 & 850 & 946.2 \\
\hline lin. comb. & $f_{s}-f_{\text {trb }}$ & $f_{\text {trb }}$ & $f_{s}$ & $2 f_{\text {trb }}$ & $f_{s}+f_{\text {trb }}$ & $3 f_{\text {trb }}$ & $3 f_{s}-f_{\text {trb }}$ & $2 f_{s}+f_{\text {trb }}$ & $3 f_{s}$ & $5 f_{\text {trb }}$ \\
& $2 f_{\text {trb }}-f_{s}$ & & $3 f_{\text {trb }}-f_{s}$ & $4 f_{\text {trb }}-f_{s}$ & & $2 f_{s}$ & $5 f_{\text {trb }}-f_{s}$ & $4 f_{\text {trb }}$ & & $4 f_{s}-f_{\text {trb }}$ \\
\hline
\end{tabular}

Table II: (color online) Frequencies of first peaks of spectrum of $p(t)$ measured $\left(f_{\text {exp }}\right)$ and simulated $\left(f_{\text {sim }}\right)$ during an $F_{3}-C_{4}$ multiphonic. Integer combinations of $f_{s}$ and $f_{\text {trb }}$ corresponding to each combination tone are indicated.

Signals recorded during this multiphonic playing are periodic signals. However, the fundamental frequency of these signals is an octave below the played note, at $f_{t r b} / 2$.

The radiated pressure signals $p_{\text {ext }}$ of the recorded [MM1, ] and the simulated [MM2, ] multiphonic are both heard as chords rather than as a single note. The playing and singing frequencies appear, along with other notes, notably the $F_{2}$ one octave below $f_{t r b}$. Though, informal listening tests highlights some perceptive differences. First, the sung note is heard louder in the experimental recording than in the simulation. Then, while listening to the recording of the musical performance, a third note, namely an $A_{4}$, can be heard, whose fundamental frequency is $f_{s}+f_{t r b}$. This note cannot be clearly heard in the simulated $p_{\text {ext }}$ signal, although its frequency components are present. One reason might be the differences in spectral balance between the experiment and the simulation, which could be related to the simplicity of the forcing signal: this latter hypothesis will be investigated later in the article.

As a conclusion, the $F_{3}-C_{4}$ multiphonic studied here appears to be quite a peculiar periodic regime of oscillation. The spectral components are $f_{s}, f_{t r b}$, their harmonics, and the combination tones of frequencies $f_{C T}=q f_{\text {trb }} \pm f_{s}, q \in \mathbb{N} \cup\{-1\}$ (except negative frequencies). The simulation model, based on a self-oscillating system, sinusoidally forced to model the contribution of the singing voice, reproduces the emergence of a regime which is very similar in terms of frequency content, but with some differences in the peak amplitudes.

Though it is periodic, this regime is not a usual self-sustained oscillation of a brass instrument: the fundamental frequency is not the trombone's oscillation frequency, but a combination tone. Several harmonics are also combination tones, each one matching with several integer combinations of $f_{s}$ and $f_{t r b}$. This fact is related to this specific situation where $f_{t r b} / f_{s}$ is a rational value. The system undergoes a 3:2 synchronization (also called an internal resonance in the dynamic system terminology) which makes combination tones integer multiples of $f_{s}-f_{t b}$. It is a particular behavior of a forced self-sustained oscillator. Academic case studies presenting this situation are developed in [Nayfeh and Balachandran, 1995, p.156] with forced Van Der Pol oscillators for instance.

According to the oscillator theory, the system studied should generate a quasi-periodic oscillation when there is no internal resonance. The following part will investigate multiphonic situations with a variable singing frequency, leading to multiple situations where $f_{s} / f_{\text {trb }}$ is irrational. 


\section{III.B Variable singing frequency}

\section{III.B.1 Experiment}

The trombone player is now asked to play a multiphonic with $f_{t r b}$ as stable as possible, while decreasing his singing frequency. He starts playing the same $F_{3}-C_{4}$ multiphonic as before. Then, he lowers its singing frequency $f_{s}$ as linearly as possible, until he reaches a frequency just above $f_{t r b}$, while he keeps playing an $F_{3}$. He then holds these playing and singing frequencies for a few seconds.

Spectrograms of the resulting $p_{m}, p$ and $p_{\text {ext }}$ are shown in Fig. 8 (a), (b) and (c), respectively. Fig. 8 (a) exhibits the evolution of the $f_{s}$ and $f_{\text {trb }}$ components in the mouth. The $f_{\text {trb }}$ component is fairly stable all along the measurement. The singing component remains stable between 0 and 5 seconds, then decreases between 5 and 10 seconds, and stays 152 musical cents above $f_{\text {trb }}$ until the end $\left(f_{s} / f_{\text {trb }}=1.09\right)$. After $t=6 \mathrm{~s}$, a new spectral component emerges in the mouth, at a frequency growing towards $f_{\text {trb }}$. The peak at $f_{s}$ has the highest amplitude all along the measurement.

The phenomenon that appears during the first 6 seconds of the spectrogram in Fig. 8 (b) and (c) is very similar to the one appearing after $12 \mathrm{~s}$ in Fig. 4 . Then, when $f_{s}$ decreases, some frequency peaks seem to "split" in two components, one with a decreasing frequency, the other one with an increasing frequency. While the frequencies get closer to one another, other components with increasing or decreasing frequencies become stronger in amplitude, collecting towards $f_{\text {trb }}$ and its harmonics. This leads to a quite rich spectrum with several secondary peaks around $f_{\text {trb }}$ and its harmonics, after $t=8 \mathrm{~s}$ when the singing frequency stabilizes.

To follow the evolution of the components more easily, spectra are computed at each second on the mouthpiece signal and plotted in Fig. 9. The splitting of certain frequency components is noticeable: for illustration, the first peak at $t=3.12 \mathrm{~s}$ at $85 \mathrm{~Hz}$ (Fig. 9 (a)) splits progressively into two distinct peaks of frequency $75 \mathrm{~Hz}$ and $100 \mathrm{~Hz}$, respectively, at $t=5.06 \mathrm{~s}$ (Fig. 9 (c)). The last spectrum, at $t=10.12 \mathrm{~s}$ (Fig. $9(\mathrm{~h})$ ), shows numerous peaks on either side of $f_{\text {trb }}$ and its harmonics.

\section{III.B.2 Simulation}

The same simulation model as in Section III.A is used to reproduce this second experiment. Starting with the same parameter values as in the previous section $\left(f_{l}=140 \mathrm{~Hz}, p_{m}^{0}=4500 \mathrm{~Pa}\right.$, $p_{m}^{1}=1350 \mathrm{~Pa}, f_{s}=282.7 \mathrm{~Hz}$ i.e. a fifth above $\left.f_{t r b}\right)$, the singing frequency is decreased linearly (sweep signal) between $t=6 \mathrm{~s}$ and $t=12 \mathrm{~s}$, to 152 cents above the playing frequency: $p_{b}(t)$ follows Eq. (3) with $f_{1}=282.7 \mathrm{~Hz}, f_{2}=206 \mathrm{~Hz}$ and $d=6 \mathrm{~s}$. The final frequency is chosen to stick with the experiment, where the final singing frequency of the musician is also 152 cents above the playing frequency. During the last two seconds, $f_{s}=206 \mathrm{~Hz}$. Just as above, spectrograms of $p_{m}$, $p$ and $p_{\text {ext }}$ are shown in Fig. 10 (a), (b) and (c), respectively. Fig. 11 represents the spectra of $p$ computed each second. Fig. 10 and 11 for simulation are equivalent to Fig. 8 and 9 for experiment. According to Eq. (3), the mouth pressure only contains the forcing term, providing a convenient view on the evolution of $f_{s}$. The first seconds of the $p$ and $p_{\text {ext }}$ spectrograms of Fig. 10 (b) and (c) are very similar to the spectrograms of Fig. 6 with components of $f_{s}$ and $f_{\text {trb }}$ plus the combination tones. Then, when $f_{s}$ starts decreasing, each component at $f_{s}$ or its harmonics splits into two components moving away from one another, one having a decreasing frequency and the other having an increasing frequency. The amplitude of increasing-frequency components is weaker than that of decreasing-frequency components. Between $t=7 \mathrm{~s}$ and $t=8 \mathrm{~s}$, a regime briefly emerges at $38,5 \mathrm{~Hz}=f_{\text {trb }} / 5$ and its harmonics, while $f_{s}=265 \mathrm{~Hz}$ : it can benoticed under the form of evenly spaced lines (at $38.5 \mathrm{~Hz}, 77 \mathrm{~Hz}, 115.5 \mathrm{~Hz}, 154 \mathrm{~Hz}, 192.5 \mathrm{~Hz}, 231 \mathrm{~Hz}$ etc.) in Fig. 10 (b) (between the dash-dotted lines) and (c). This is a periodic regime due to a $5: 1$ internal resonance. 
Indeed, at this point the $f_{s} / f_{\text {trb }}$ ratio is rational and the resulting regime is periodic. The resulting frequency is very close to the first acoustic resonance frequency of the trombone $(38.9 \mathrm{~Hz})$ so the first mode may sustain this oscillation. This will be addressed in Section III.C. After $t=10 \mathrm{~s}$, the existing components strengthen and new ones appear, to end up with several secondary peaks on each side of the components of the auto-oscillation. These new peaks are particularly visible in the last spectra in Fig. 11.
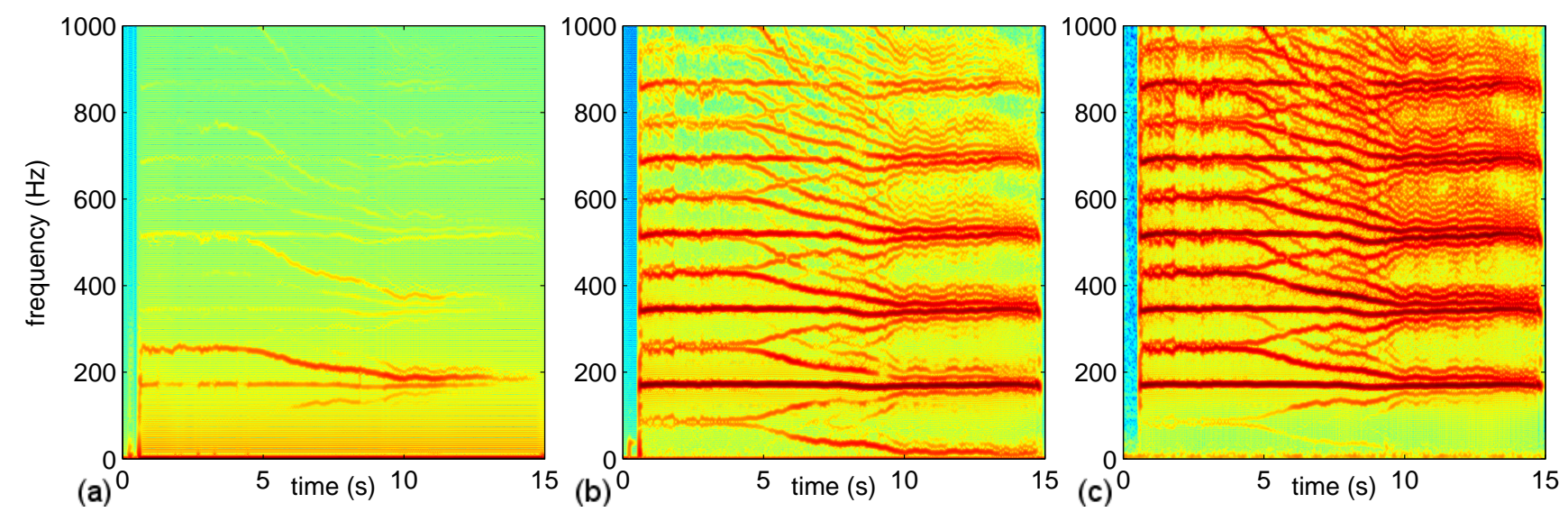

Figure 8: (color online) Experiment: Spectrograms of $p_{m}(\mathrm{a}), p(\mathrm{~b})$ and $p_{\text {ext }}(\mathrm{c})$ measured during a multiphonic. Hanning window of width $0.2 \mathrm{~s}$, overlapping of $95 \%$, zero-padding of the signal until frequency precision is under $1 \mathrm{~Hz}$. The singing frequency is constant at $f_{s}=255 \mathrm{~Hz}$ (note $C_{4}$ ) for 5 seconds, then decreases towards $f_{s}=185 \mathrm{~Hz}$ (slightly above $f_{\text {trb }}$, note $F_{3}$ ) and remains at this value after $t=10 \mathrm{~s}$. The playing frequency remains as constant as possible, with $f_{\text {trb }} \in[167.2: 173.4]$.
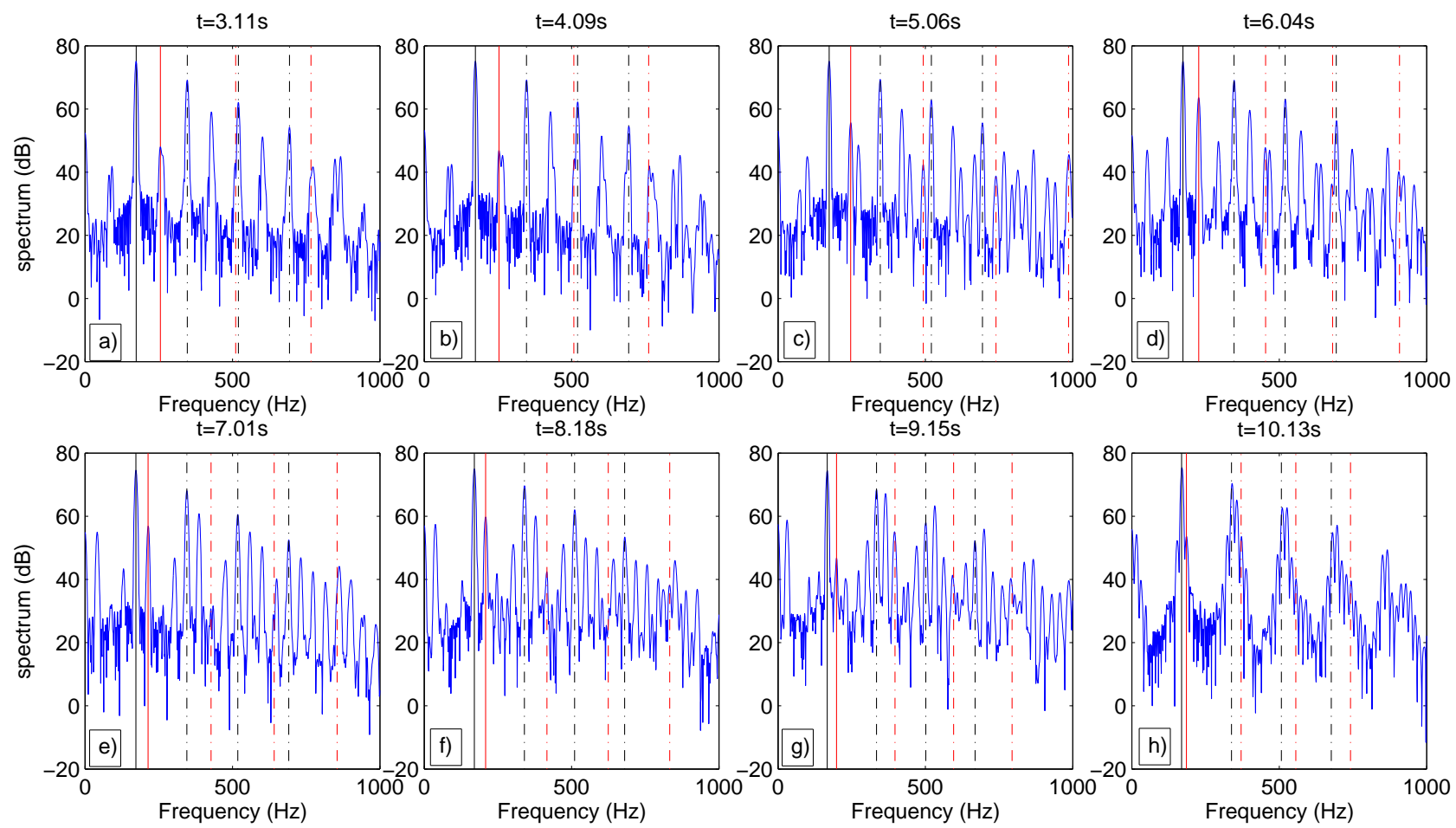

Figure 9: (color online) Experiment: Spectra taken from instants of Fig. 8b) each second between approximately $t=3 \mathrm{~s}$ and $t=10 \mathrm{~s}$, when $f_{s}$ decreases. The plain lines represent $f_{\text {trb }}$ (black) and $f_{s}$ (red), the dash-dotted lines represent their respective harmonics. 

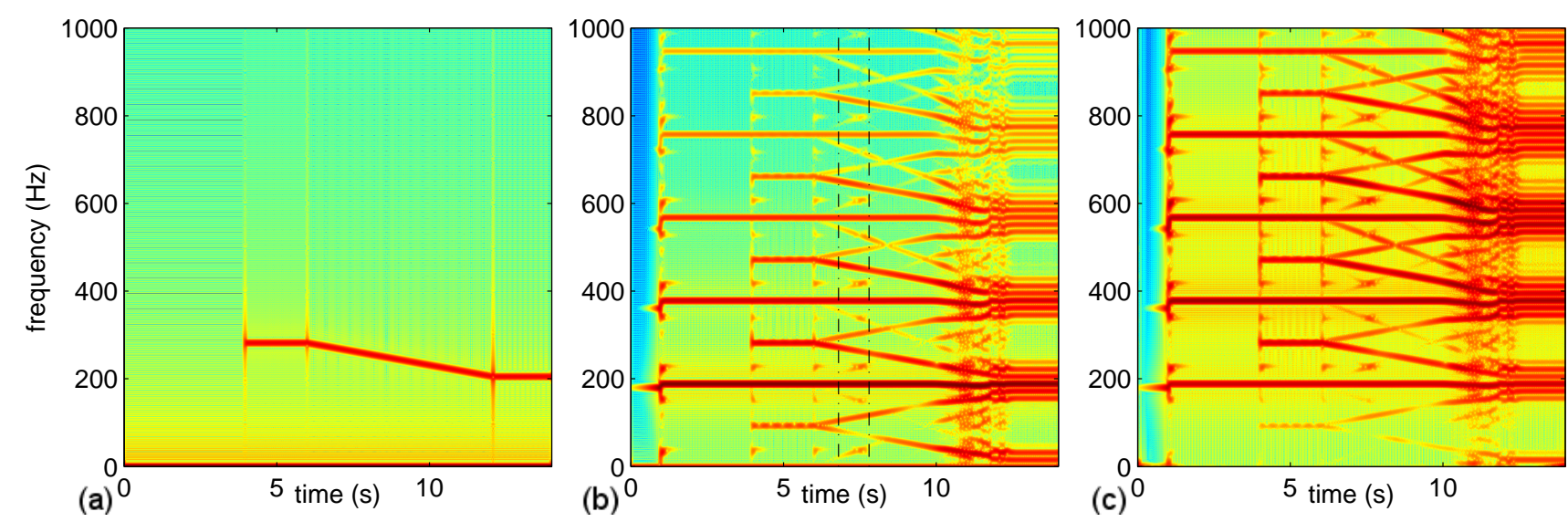

Figure 10: (color online) Simulation: Spectrograms of the simulated $p_{m}$ (a), $p$ (b) and $p_{\text {ext }}$ (c). Hanning window of width $0.2 \mathrm{~s}$, overlapping of $95 \%$, zero-padding of the signal until frequency precision is under $1 \mathrm{~Hz}$. The forcing term appears after $t=4$ seconds at $f_{s}=282.7 \mathrm{~Hz}$ (a fifth above $\left.f_{\text {trb }}\right)$, then is steady until $t=6 \mathrm{~s}$; then decreases linearly towards $f_{s}=206 \mathrm{~Hz}(155$ cents above $f_{\text {trb }}$ ) for 6 seconds: then it stays at this frequency for 2 seconds. The playing frequency remains stable at $f_{t r b}=188.7 \mathrm{~Hz}$ all along the simulation. Vertical dash-dotted lines in (b) highlight the periodic oscillation regime at $38.5 \mathrm{~Hz}$.
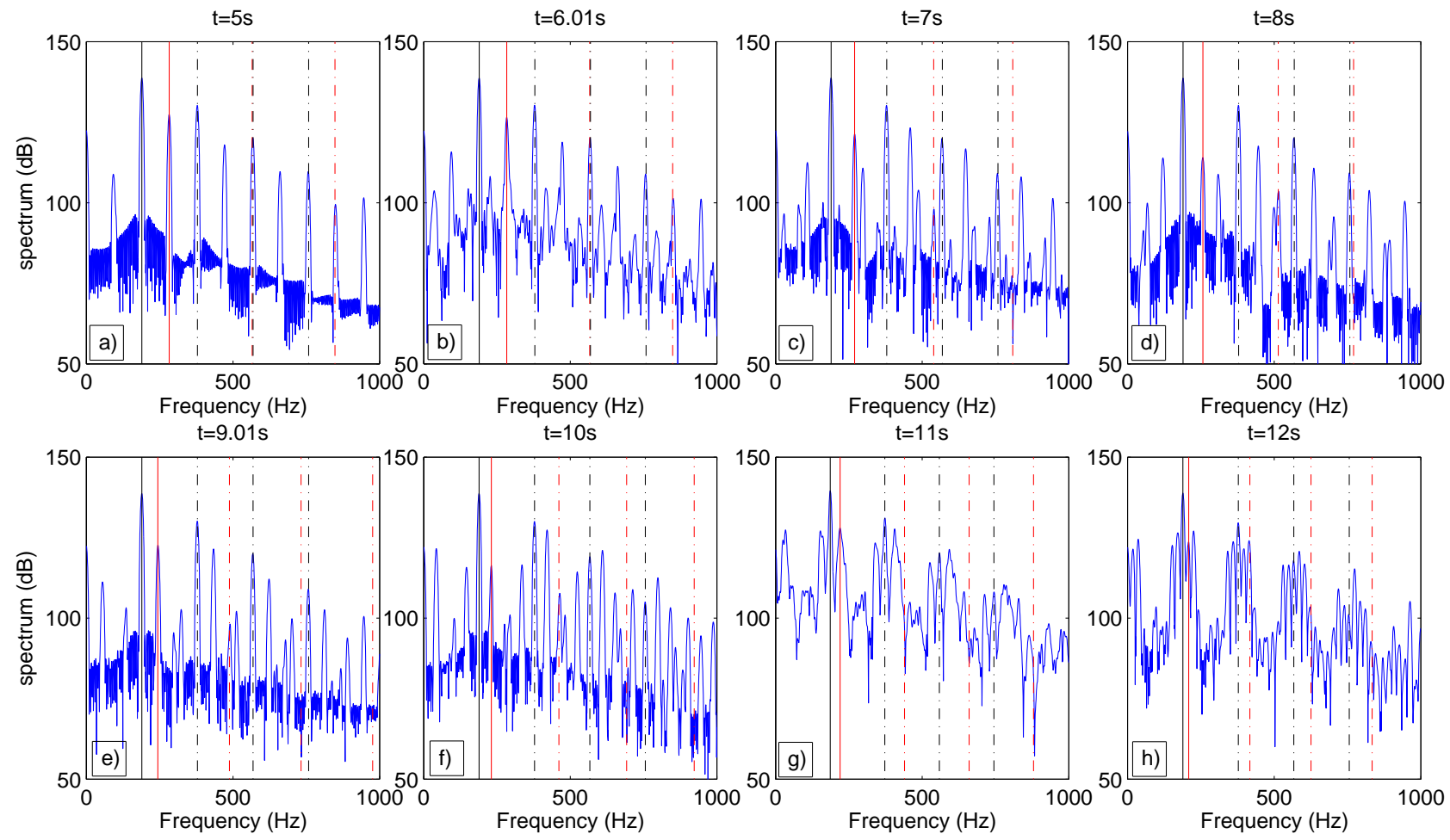

Figure 11: (color online) Simulation: Spectra of the simulated $p$, taken from the spectrogram in Fig. 10b) each second, between approximately $t=5 s$ and $t=12 s$ where $f_{s}$ decreases. The plain vertical lines represent $f_{t r b}$ and $f_{s}$, the dash-dotted lines represent their respective harmonics.

\section{III.B.3 Discussion}

As in the previous experiment, several frequency components appear to be harmonics of neither $f_{t r b}$ nor $f_{s}$. At the beginning, the frequencies are the same as in Section III.A. Certain integer 
combinations result in the same combination tones, like for example $3 f_{\text {trb }}=2 f_{s}$. Then, when $f_{s}$ decreases, these integer combinations are no longer equal since $f_{s}$ and $f_{\text {trb }}$ are no longer in a $3: 2$ ratio: the combination tone frequencies either increase or decrease, depending on the sign in front of $f_{s}$ in the integer combination. The frequencies contained in the signal are no longer integer multiples of the lowest frequency. The oscillation becomes quasi-periodic as soon as $f_{s} / f_{\text {trb }}$ is no longer rational.

Frequencies of the components of $p$ during the phase where $f_{s}$ decreases are recorded, and plotted with marks in Fig. 12 (a) (experimental measurement) and (b) (simulation). Some integer combinations of $f_{s}$ and $f_{t r b}$, of positive frequency $q f_{t r b} \pm f_{s}, q \in \mathbb{N} \cup\{-1\}$ are also plotted with plain lines on the same figures. The match between each peak frequency and one integer combination is remarkable, with a maximum relative error of $2.5 \%$. Therefore, the emerging frequency components are integer combinations of $f_{s}$ and $f_{\text {trb }}$, just as the $F_{3}-C_{4}$ multiphonic. It can be concluded that this more complex multiphonic regime is due to the same phenomenon as in Section III.A, emerging from the coupling between a self-sustained oscillator and a forcing term. However, here the oscillation regime is quasi-periodic.
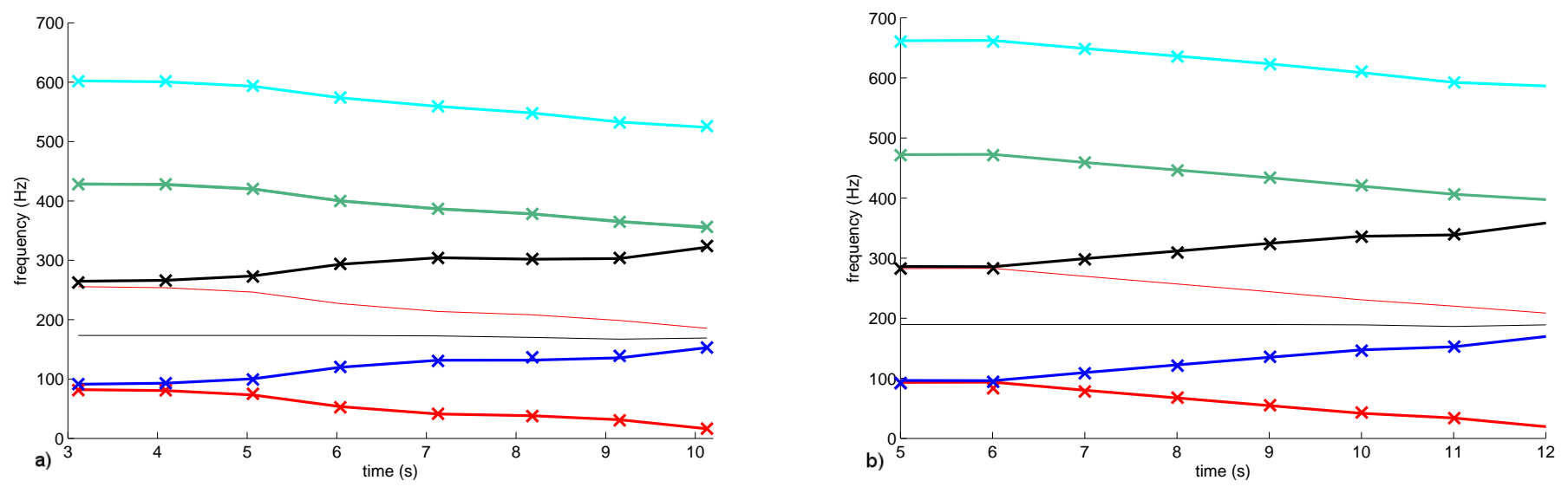

Figure 12: (color online) Plot of $f_{s}$ (red), $f_{t r b}$ (black) and some integer combinations (plain lines) along with the frequencies of the five first components of experimental (a) and simulation (b) mouthpiece pressure (marks). These marks correspond to the peak frequencies in Fig. 9 and 11. The recorded values very closely match the integer combinations.

The simulated $p_{\text {ext }}$ and the musical performance were heard as similar during the experiment, yet differences exist. In the recorded multiphonic [MM3, ], an $A_{3}$ can be heard at the beginning. When $f_{s}$ begins to change, this $A_{3}$ rapidly decreases in loudness. During the decrease in $f_{s}$, certain notes become audible while others disappear. These notes can also be heard in the simulated $p_{\text {ext }}$ [MM4, ] but their overall loudness is weaker. At the end of this multiphonic, $f_{s}$ and $f_{\text {trb }}$ are close to each other. There, a "rolling" or "beating" sound can be heard, both in the experimental and in the simulated $p_{\text {ext }}$ signals. This could be explained by the very low $f_{s}-f_{\text {trb }}=16 \mathrm{~Hz}$ component $(16.3$ $\mathrm{Hz}$ in measurements, $17.3 \mathrm{~Hz}$ in simulation), perceived as a modulation of the sound.

These observations can be related to the spectral envelopes of the measured and the simulated $p$ : comparatively with the peaks at $f_{t r b}$, the combination tones and the harmonics of $f_{s}$ have a smaller amplitude in simulation than in experimental measurements. The $f_{s}$ component of $p$ is an exception as it is weaker in measurement than in simulation. At the end of the multiphonic, the peaks at harmonics of $f_{t r b}$ are flanked by smaller peaks on both sides. These secondary peaks are significantly weaker in measurement than in simulations.

It can be assumed that these amplitude differences between experiment and simulation are related to the sinusoidal nature of the forcing whereas the singing is a more complex periodic signal. To 
assess this hypothesis, another simulation is carried out, this time with a forcing signal reproducing the three first harmonics of the recorded singing signal. The oscillating component of $p_{m}$ now consists of three sinusoids, with the same relative amplitudes and phases as the first components of the measured sung signal. Figure 13 compares spectra of $p$ simulated with a forcing signal consisting of one or three harmonics (blue and red plots, respectively), at the begining (Fig. 13 (a) and the end (Fig. 13 (b) of a simulation with decreasing $f_{s}$. All parameters of the three-harmonic forcing simulation are equal to those of the simulation presented in Fig. 10 and Fig. 11 except the $p_{m}$ signal.
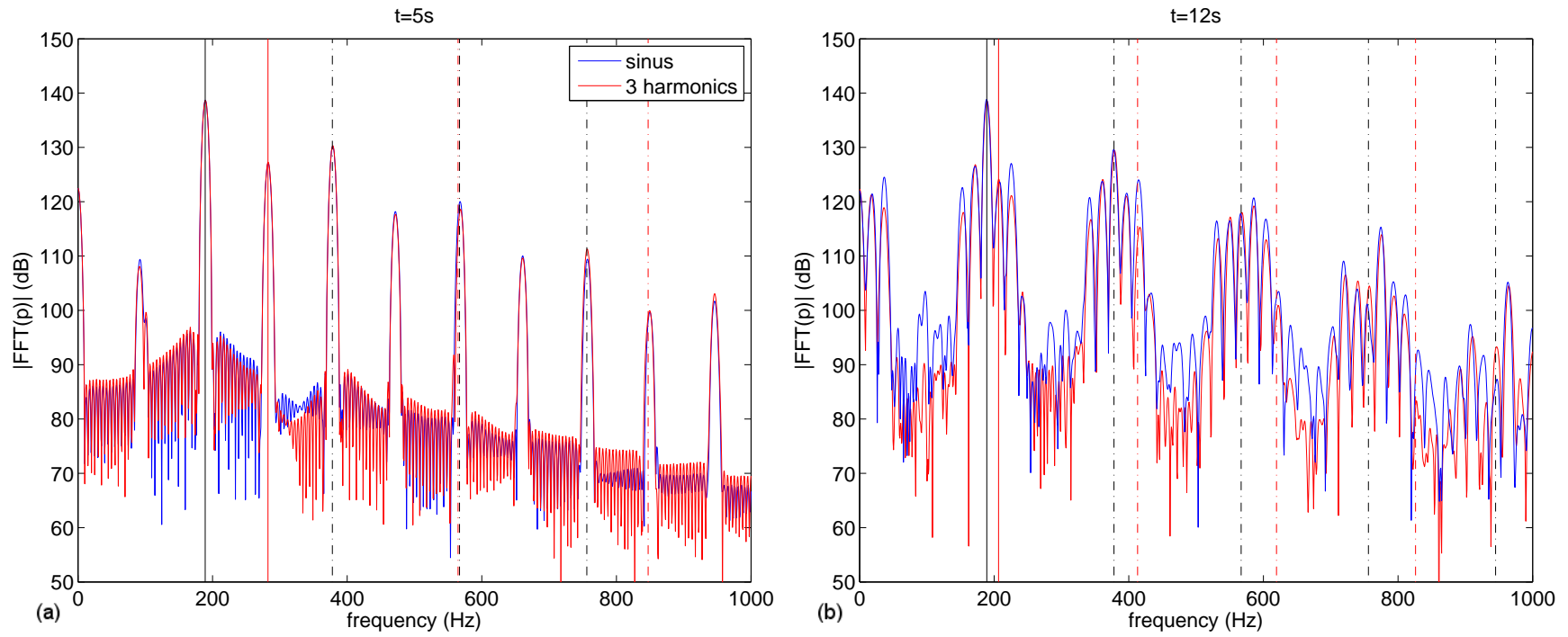

Figure 13: (color online) Comparison of spectra of internal pressures simulated either with a sinusoidal forcing component, or a 3-harmonic forcing component. Hanning window of width 0.2 $\mathrm{s}$, zero-padding of the signal until frequency precision is under $1 \mathrm{~Hz}$. Frequency decreases as in Fig. 10. Fig. (a) is the spectra of $p$ at the beginning of the simulations, Fig. (b) at the end. Differences in terms of amplitude are small in (a) and reach $6 \mathrm{~dB}$ in (b). Vertical lines indicate $f_{\text {trb }}$ and $f_{s}$ (plain) and their harmonics (dash-dotted).

Adding harmonics to the forcing signal only results in minor changes in the spectral envelope of the signal. Apart from the components at $2 f_{s}$ and $3 f_{s}$ being logically stronger, the major difference between these simulations is the relative height of the peaks as compared to the numerical noise. Between amplitude peaks, the minima are visibly weaker with a three-component forcing signal, particularly at the end of the simulation (Fig.13 (b)). The influence on the amplitude of combination tones is not significant. The lack of major difference could be explained by the weakness of the harmonics of the forcing, the second and third harmonics of $f_{s}$ being respectively $17.5 \mathrm{~dB}$ and $27.5 \mathrm{~dB}$ weaker than the fundamental.

From the results on multiphonics with a sliding $f_{s}$ described in this section the conclusions from the previous section can be generalized: the self-oscillating model with an additional forcing term is sufficient to simulate multiphonics, resulting in the same frequency components as the measured ones. The frequencies of the components of both the simulated and the measured $p$ match very well with integer combinations of the instrument self-oscillation frequency and the singing or forcing frequency: these components are either harmonics of $f_{s}$ and $f_{t r b}$ or combination tones. This consolidates the idea that multiphonic regimes of brass instruments are either quasi-periodic regimes or, when the $f_{\text {trb }} / f_{s}$ ratio is rational, periodic regimes. Both behaviors come from the same phenomenon. The $F_{3}-C_{4}$ multiphonic considered in III.A is a particular case, where all 
frequencies in the signal are harmonic because of the 3:2 internal resonance.

The simulation model tested here reproduces this behavior accurately, in spite of the simplicity of the model. The main difference is the spectral envelope. A forcing signal closer to the singing signal does not significantly change this limitation.

\section{III.C Simulations with one acoustic resonance}

New simulations with an even simpler model are carried out. Not only the forcing is sinusoidal as seen in Fig. 14, but the resonator is reduced to one resonance, the third acoustic mode of the trombone - the one used for playing an $F_{3}$.

A simulation with a decreasing sinusoidal forcing is carried out. The playing frequency with this resonator is $f_{t r b}=180.5 \mathrm{~Hz}$, so the playing frequency decreases from $f_{s}=270 \mathrm{~Hz}$ to $f_{2}=196.4$ $\mathrm{Hz}$ for 6 seconds to keep the same frequency ratios as in Section III.B.
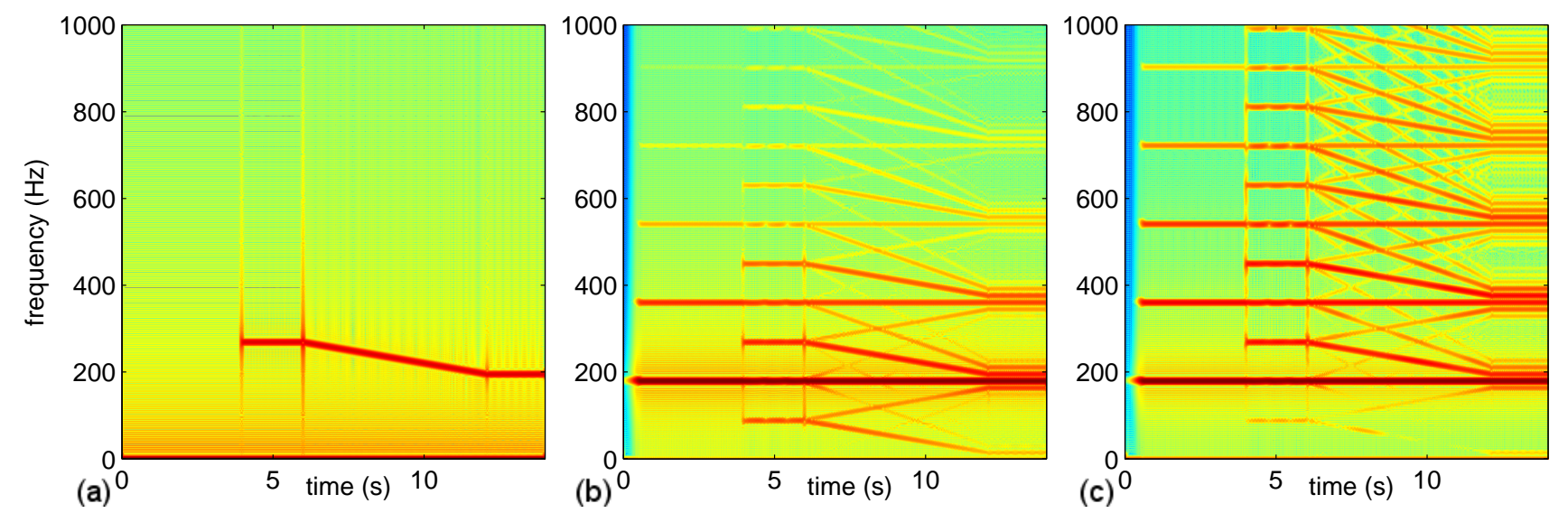

Figure 14: (color online) Simulation: Spectrograms of $p_{m}, p$ and $p_{\text {ext }}$ simulated with a one-mode resonator. Hanning window of width $0.2 \mathrm{~s}, 95 \%$ overlapping, zero-padding of the signal until frequency precision is under $1 \mathrm{~Hz} . f_{\text {trb }}=180.5 \mathrm{~Hz}, f_{s}$ from $270 \mathrm{~Hz}$ to $196.4 \mathrm{~Hz}$. The results are comparable with those presented in Fig. 10 with weaker components at high frequencies.

The frequency components of the internal pressure $p$ are harmonics of $f_{\text {trb }}$ and $f_{s}$ or combination tones, as in the previous simulations and measurements, for all values of $f_{s}$ tested. Thus, the multiphonic behavior appears with this model. However, the amplitude of most frequency components is weaker. This is illustrated by the amplitude differences reported in Table III: while the amplitude of the component at $f_{\text {trb }}$ remains fairly constant, all other components are significantly weaker for the 1-mode simulation. Harmonics of $f_{t r b}$ are no longer supported by the modes 6,9 and 12 , which affects the amplitude of combination tones. The $f_{s}$ component in $p(t)$ is weaker, even though the amplitude of the forcing is equal: the modulus of the resonator impedance is 13 times weaker at $f_{s}$ with only one mode than with 13 modes, which reduces the amplitude of the $f_{s}$ component in the mouthpiece. The lowest component $f_{s}-f_{\text {trb }}=15.9 \mathrm{~Hz}$ at the end of the simulation is also weaker, but it is perceived as a modulation of the sound, making a "rolling" sound just like in previous simulations and in measurement. The brief regime at fundamental frequency $f_{\text {trb }} / 5$ which can be seen between $t=7 \mathrm{~s}$ and $t=8 \mathrm{~s}$ in Fig 10 does not occur here, because of the absence of the first mode. Another simulation with all acoustic modes except the first one does not make this regime appear, which strengthens this hypothesis. This simulation result is not shown here for the sake of brevity. 


\begin{tabular}{|c||c|c|c|c|c|c|c|}
\hline frequency & $f_{s}-f_{\text {trb }}$ & $f_{\text {trb }}$ & $f_{s}$ & $2 f_{\text {trb }}$ & $f_{s}+f_{\text {trb }}$ & $3 f_{\text {trb }}$ & $3 f_{s}-f_{\text {trb }}$ \\
\hline difference $(\mathrm{dB})$ at $t=5 \mathrm{~s}$ & 0.6 & 0.3 & 10.7 & 27 & 11.7 & 23.2 & 16 \\
difference $(\mathrm{dB})$ at $t=9 \mathrm{~s}$ & 7.6 & 0.5 & 4.8 & 27.4 & 14.2 & 27 & 8.7 \\
difference $(\mathrm{dB})$ at $t=13 \mathrm{~s}$ & 26 & 1.2 & 4.5 & 17.9 & 12.7 & 25.1 & 17.4 \\
\hline
\end{tabular}

Table III: Differences (in dB) in the amplitudes of the first peaks for one-mode and 13-mode simulations, measured on the data displayed in Fig. 14, at three time points: $t=5 \mathrm{~s}$ when $f_{s}=270$ $\mathrm{Hz}, t=9 \mathrm{~s}$ when $f_{s}=233 \mathrm{~Hz}$ and decreases, and $t=13 \mathrm{~s}$ when $f_{s}=196.4 \mathrm{~Hz}$. While amplitudes of the peaks at $f_{\text {trb }}$ are equivalent, other components are much weaker when the resonator has a single mode.

The quasi-periodic regime related to multiphonics therefore occurs even with a very simple instrument model: a one-DOF mechanical exciter non-linearly coupled with a one-mode acoustic resonator, self-oscillating and forced with a sinusoidal signal.

\section{CONCLUSIONS}

Both measurement and simulation results presented in this paper confirm the type of oscillating regime of the multiphonic sounds studied. When the musician sings and plays different notes simultaneously, the resulting pressure signal inside the instrument contains harmonics of the sung and played frequencies, as well as combination tones, whose frequencies are integer combinations of $f_{s}$ and $f_{t r b}$. This is verified not only in the particular case of an $F_{3}-C_{4}$ multiphonic as in Section III.A but also in the case of a time-variable frequency. This generally corresponds to quasi-periodic oscillation, except when an internal resonance occurs and the ratio between playing and singing frequencies is rational. In this latter case, the oscillation is periodic, though it is different from the usual periodic self-oscillation of a brass instrument.

To some extent, the trombone physical model used in this paper is able to simulate this phenomenon. Even if the simulated and measured $f_{t r b}$ are not exactly the same (as usual with an outward-striking lip model), the frequency content of the simulated internal pressure of the instrument $p$ is very similar to the probe microphone measurements inside the mouthpiece, with harmonics of both the sung and played signals along with combination tones. This similarity also applies when $f_{s}$ decreases with time, and when the instrument model is simplified at most. The major difference between simulation and measurement consists in differences in the spectral envelopes: the amplitude of the peaks corresponding to combination tones is generally weaker in measurement than in simulation when the forcing is sinusoidal. This is particularly true when $f_{s}$ and $f_{\text {trb }}$ are close to each other, the secondary peaks being much weaker in measurement. A richer forcing signal with three harmonics, closer to the measured sung signal, does not dramatically change the results: changing the forcing signal does not seem the best way to improve the simulation.

While the simulated blowing pressure $p_{m}^{0}$ is of the same order of magnitude as blowing pressures usually measured on trombone players [Bouhuys, 1968, Fréour and Scavone, 2013], the amplitude of the forcing signal $p_{m}^{1}$ used in our simulations is about 15 times higher than the amplitude measured in the recorded mouth pressure signal $p_{m}$. This is the main limitation of this model for multiphonic simulation. Yet, these very different input parameters give comparable results in terms of internal pressure $p$, the difference in amplitude between the peaks at $f_{\text {trb }}$ and $f_{s}$ being of the same order of magnitude in the measured and simulated $p$ spectra. This is the main limitation of our model. A more complex model, taking into consideration the couplings between the lips, the vocal folds, the resonances in the instrument and those in the vocal tract would possibly give 
a better simulation of multiphonics in terms of amplitude of the frequency components, yet at the expense of a far greater complexity.

\section{ACKNOWLEDGMENTS}

Thanks to Maxime Demartin, trombonist, for obligingly performing the required exercises on our experimental device, and entrusting his trombone to us for impedance measurements. Thanks to the technical staff of the SERM, Olivier Pot and Vincent Long, for their help in designing and building the experimental setup. Thanks to Patrick Sanchez and Thibault Lefranc for their help for input impedance and radiation transfer function measurements. Thanks to Jean Kergomard and Pierre Vigué for their interesting discussions about this article.

This work has been carried out in the framework of the Labex MEC (ANR-10-LABX-0092) and of the A*MIDEX project (ANR-11-IDEX-0001-02), funded by the « Investissements d'Avenir » French Government program managed by the French National Research Agency (ANR).

\section{References}

[Backus, 1978] Backus, J. (1978). Multiphonic tones in the woodwind instruments. J. Acoust. Soc. Am, 32(2):591-599.

[Blanc et al., 2010] Blanc, F., de la Cuadra, P., Fabre, B., Castillo, G., and Vergez, C. (2010). Acoustics of the Flauta de Chinos. In Proceedings of the $20^{\text {th }}$ International Symposium on Music Acoustics, paper no. 19, pages 1-5, Sydney and Katoomba, Australia.

[Bouhuys, 1968] Bouhuys, A. (1968). Pressure-flow events during wind instrument playing. Annals of New York Academy of Sciences, 155(1):264-275.

[Campbell, 2004] Campbell, M. (2004). Brass instruments as we know them today. Acta Acustica united with Acustica, 90:600-610.

[Campbell and Greated, 1994] Campbell, M. and Greated, C. (1994). The musician's guide to acoustics. J.M. Dent \& sons Ltd., London, 613 pages.

[Castellengo, 1981] Castellengo, M. (1981). Sons multiphoniques aux instruments à vent (multiphonic sounds on woodwind instruments). Rapports de l'IRCAM.

[Castellengo et al., 1983] Castellengo, M., Caussé, R., and Sluchin, B. (1983). étude acoustique de l'émission multiphonique aux cuivres (acoustical study of multiphonic emission on brass instruments. In Proceedings of International Conference on Acoustics 1983, pages 355-385, Paris-Lyon-Toulouse.

[Chaigne and Kergomard, 2016] Chaigne, A. and Kergomard, J. (2016). Acoustics of musical instruments. Springer-Verlag, New York, 869 pages.

[Chen et al., 2012] Chen, J.-M., Smith, J., and Wolfe, J. (2012). Do trumpet players tune resonances of the vocal tract? J. Acoust. Soc. Am., 131(1):722-727.

[Cullen et al., 2000] Cullen, J., Gilbert, J., and Campbell, M. (2000). Brass instruments: Linear stability analysis and experiments with an artificial mouth. Acta Acustica united with Acustica, $86: 704-724$. 
[Dalmont et al., 1995] Dalmont, J., Gazengel, B., Gilbert, J., and Kergomard, J. (1995). Some aspects of tuning and clean intonation in reed instruments. Applied Acoustics, 46(1):19-60.

[Doc et al., 2014] Doc, J., Vergez, C., and Missoum, S. (2014). A minimal model of a single-reed instrument producing quasi-periodic sounds. Acta Acustica united with Acustica, (3):543-554.

[Eliott and Bowsher, 1982] Eliott, S. and Bowsher, J. (1982). Regeneration in brass instruments. Journal of Sound and Vibration, 83:181-217.

[Fletcher, 1993] Fletcher, N. (1993). Autonomous vibration of simple pressure-controlled valves in gas flows. J. Acoust. Soc. Am., 93(4):2172-2180.

[Fréour and Scavone, 2013] Fréour, V. and Scavone, G. (2013). Acoustical interaction between vibrating lips, downstream air column, and upstream airways in trombone performance. $J$. Acoust. Soc. Am., 134(5):3887-3898.

[Gibiat and Castellengo, 2000] Gibiat, V. and Castellengo, M. (2000). Period doubling occurences in wind instruments musical performance. Acta Acustica united with Acustica, 86:746-756.

[Hezard et al., 2014] Hezard, T., Freour, V., Caussé, R., Helie, T., and Scavone, G. (2014). Synchronous multimodal measurements on the lips and glottis: comparison between two humanvalve oscillating systems. Acta Acustica united with Acustica, 100:1172-1185.

[Hirschberg et al., 1996] Hirschberg, A., Gilbert, J., Msallam, R., and Wijnands, P. (1996). Shock waves in trombones. J. Acoust. Soc. Am., 99:1754.

[Hirschberg et al., 1995] Hirschberg, A., Kergomard, J., and Weinreich, G. (1995). Mechanics of musical instruments. Springer-Verlag, Wien, Austria, 369 pages.

[Iniacio et al., 2008] Iniacio, O., Antunes, J., and Wright, M. (2008). Computational modelling of string-body interaction for the violin family and simulation of wolf notes. Journal of Sound and Vibration, (310):260-286.

[Keefe and Laden, 1991] Keefe, D. and Laden, B. (1991). Correlation dimension of woodwind multiphonic tones. J. Acoust. Soc. Am., 90(4):1754-1765.

[Macaluso and Dalmont, 2011] Macaluso, C. and Dalmont, J.-P. (2011). Trumpet with nearperfect harmonicity: Design and acoustic results. J. Acoust. Soc. Am, 129(1):404-414.

[MM1, ] MM1. Link to the simulated radiated pressure with constant singing frequency, stored online.

[MM2, ] MM2. Link to the measured radiated pressure with constant singing frequency, stored online.

[MM3, ] MM3. Link to the measured radiated pressure with variable singing frequency, stored online.

[MM4, ] MM4. Link to the simulated radiated pressure with vairable singing frequency, stored online.

[Myers et al., 2012] Myers, A., Pyle, R., Gilbert, J., Campbell, M., Chick, J., and Logie, S. (2012). Effects of nonlinear sound propagation on the characteristic timbres of brass instruments. $J$. Acoust. Soc. Am., 131(1):678-688. 
[Nayfeh and Balachandran, 1995] Nayfeh, A. and Balachandran, B. (1995). Applied nonlinear dynamics: Analytical, computational and Experimental methods. Wiley-VCH Verlag KGaA, Weinheim, 700 pages.

[Silva, 2009] Silva, F. (2009). Émergence des auto-oscillations dans un instrument de musique à anche simple (Emergence of self-sustained oscillations in a single reed music instrument). $\mathrm{PhD}$ thesis, Universite de Provence Aix-Marseille 1.

[Silva, 2013] Silva, F. (2013). Source website of the moreesc software. http://moreesc.lma. cnrs-mrs.fr/. Accessed: 2015-06-22.

[Silva et al., 2007] Silva, F., Kergomard, J., and Vergez, C. (2007). Oscillation thresholds for "striking outwards" reeds coupled to a resonator. In Proceedings of ISMA 2007, Barcelona.

[Sluchin, 1995] Sluchin, B. (1995). Jeu et chant simultanés sur les cuivres (Playing and singing simultaneously on brass instruments). Editions Musicales Européennes, 127 rue Amelot, 75011 Paris, 25 pages.

[Terrien et al., 2013] Terrien, S., Vergez, C., Cuadra, P. D. L., and Fabre, B. (2013). Is the jetdrive flute model able to produce modulated sounds like flautas de chinos? In Proceedings of the Stockholm Music Acoustics Conference, pages 465-472, Stockholm.

[Velut et al., 2016] Velut, L., Vergez, C., Gilbert, J., and Djahanbani, M. (2016). How well can linear stability analysis predict the behavior of an outward valve brass instrument model? hal-01245846; Submitted to Acta Acustica united with Acustica, currently in revision: https://hal.archives-ouvertes.fr/hal-01245846.

[von Helmholtz, 1877] von Helmholtz, H. (1877). On the Sensations of Tone. Dover Publications Inc., New York, 608 pages.

[Wolfe and Smith, 2008] Wolfe, J. and Smith, J. (2008). Acoustical coupling between lip valves and vocal folds. Acoustics Australia, 36(1):23-27.

[Wright and Campbell, 1998] Wright, H. and Campbell, M. (1998). Analysis of the sound of chilean pifilca flutes. The Galpin Society Journal, 51:51-63.

[Yoshikawa, 1995] Yoshikawa, S. (1995). Acoustical behavior of brass player's lips. J. Acoust. Soc.Am, 97(3):1929-1939. 